\title{
CATACLYSMIC VARIABLE STARS
}

\author{
BRIAN WARNER \\ Department of Astronomy, University of Cape Town, Rondebosch 7700. \\ South Africa
}

\section{Introduction}

The evolution of single stars on and away from the main sequence is well understood A degenerate core is formed in a star as the star leaves the main sequence and expands to a giant with a radius typically $50-500 \mathbf{K}_{0}$. Observationally it is known that most stars are members of binary systems, and among these many have orbital periods less than $100 \mathrm{y}$. It can happen, therefore, that the expanding envelope of the primary of a binary system can reach to the secondary. As this happens, the primary fills its Roche tidal lobe and transfers matter to the secondary; if the primary has a radiative envelope the rate at which this occurs exceeds the Eddington limit of the secondary, which therefore repels the incoming gas, forming a common envelope around the two stars. Friction within the envelope causes the stars to spiral towards each other until the energy and angular momentum extracted from the binary orbit and transferred to the envelope are sufficient to eject the common envelope as a planetary nebula, leaving a short period binary $\left(P_{\text {orb }} \widetilde{<} 1 \mathrm{~d}\right.$ ) comprising a white dwarf and a main sequence star.

This mechanism of producing short period binaries containing white dwarfs, proposed by Ostriker and by Paczynski (1976), is the probable origin of the class of objects known as Cataclysmic Variable Stars (CVs), which encompass the classical novae, dwarf novae, novam like variables and a variety of related objects. Evidence has been accumulating for forty years (Crawford \& Kraft 1956, Warner 1995a) that every CV consists of a secondary star (usually a dwarf, but a few systems contain giants) filling its Roche lobe and transferring mass to a white dwarf primary. In systems of normal chemical composition the orbital periods lie between 75 mins and $\sim 250 \mathrm{~d}$, with the majority having $P_{\text {orb }} \approx<\mathrm{h}$. A few hydrogen-free systems are known for which 17 mins $<P_{\text {orb }}<50$ mins. It should be noted that $C V s$ are very compact binary systems: for $P_{\text {orb }} \widetilde{<} 2.5 \mathrm{~h}$ such a binary would fit inside the Sun.

Cataclysmic variables, interesting as they are in their own right, provide insight into the physics and phenomenology of a range of other objects. In particular, the gas flowing from the secondary creates an accretion disc around the primary, and it is in the CVs that quasi-stable and time-dependent accretion dises are observationally easiest to study, which informs interpretations of discs around young stars, in X-ray binaries and in active galactic nuclei. The low mass $\mathrm{X}$-ray binaries, with their neutron star and black hole primaries, are higher energy analogues of the CVs.

The history of $\mathrm{CV}$ research follows closely the development of astronomical technology. The rapidity with which the luminosities and spectra of CVs vary around an orbital cycle has always put strong demands on instrumentation. Early photographic photometry contributed only to the long term light curves, revealing the large amplitude variations of dwarf novae and classical novae. It was the pioneering photoelectric photometry of A.P. Linnell and M.F Walker in the 1950 s that showed that CVs vary on very short time scales, typically with variations of 0.01 to $0.2 \mathrm{mag}$ within minutes. This flickering is now recognised as a characteristic of the mass transfer process, having its origins in the shocked region where the mass transfer stream impacts onto the outer rim of the accretion disc, and in the inner regions of the disc itself. Walker's discovery of low amplitude coherent $7 \mathrm{l} \mathrm{s}$ variations in the remnant of Nova Herculis 1934 (DQ Her) (Walker 1956) was the most spectacular discovery of this era of $\mathrm{CV}$ research.

The later development of photon counting photometry, and its compatibility with on-line application of computers (Nather \& Warner 1971, Warner 1988a), reinvigorated high time resolution photometry of $\mathrm{CVs}$, leading to discovery of more examples of rapid coherent 
brightness variations and a range of quasi-periodic variations during the outbursts of dwarf novae (Warner \& Robinson 1972a).

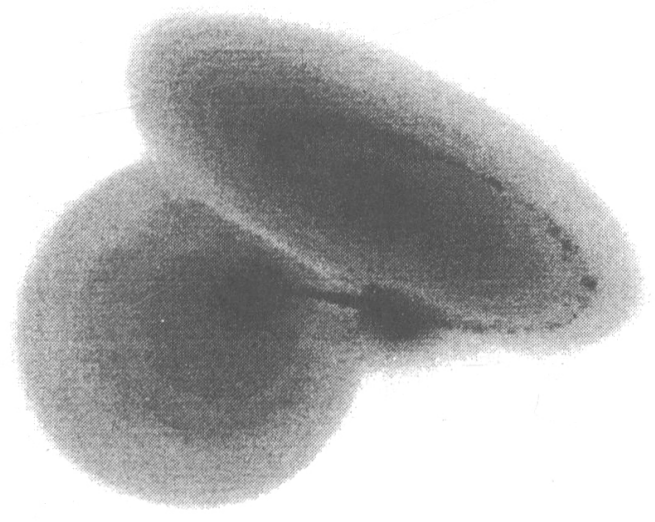

Fig. 1. Artist's impression of a cataclysmic variable star. For a colour version see the front cover of PASP Vol. 105, February 1993 has been the discovery of $\mathrm{TeV}$ gamma rays from the nova-like variable $\mathrm{AE}$ Aqr modulated with the 33 s rotation period of the primary (Meintjies et al. 1994). The same object, and a few other CVs, have been detected at radio wavelengths.

Since the discovery of strong linear and circular optical polarization in AM Her in 1976 (Tapia 1977) a further dimension has been added to CV research. Large magnetic fields $\left(10^{6}-\right.$ $\left.10^{8} \mathrm{G}\right)$ are known among field white dwarfs. The presence of strong fields in $\mathrm{CV}$ primaries, first detected through optical polarization, has a dramatic effect on the mass transfer process. If the field is sufficiently strong $\left(>10^{7} \mathrm{G}\right)$ the stream, ionized by hard radiation from the vicinity of the primary, threads onto the field lines and falls directly to the primary without forming an accretion disc. Furthermore, the interaction between the field of the primary and that of the secondary is sufficient to synchronize the primary's rotation with the orbital revolution of the secondary. Such systems are known as polars, or AM Her stars. The infalling gas passes through a standoff shock before settling onto the white dwarf. This shock-heated gas is a source of $\sim 10 \mathrm{keV}$ bremsstrahlung and polarized optical and infrared cyclotron emission. Reprocessed radiation from the surface of the white dwarf adds a $\sim 40 \mathrm{eV}$ component.

If the primary's field is weaker, synchronization generally does not happen, and an accretion disc can form, but its inner edge is determined by the region where the magnetosphere controls the final mass flow onto the primary. These objects are known as

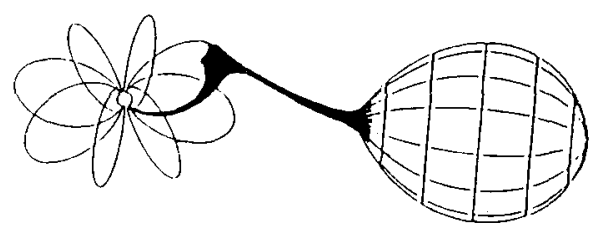

Fig. 2. Schematic view of a polar, showing the gas stream becoming attached to field lines of the primary. From Cropper (1990). intermediate polars (IPs); DQ Her and AE Aqr are examples, but in most IPs the rotation period of the primary is typically -20 mins

To these basic structures of CVs yet another dimension of behaviour is seen in their long term behaviour. The rate of mass transfer from the secondary may vary on a range of time scales. The stream itself is structured, probably through turbulent or magnetically controlled flow out of the secondary; this is seen in rapid luminosity 
variations, particularly in polars (Wood, Imamura \& Wolff 1992). Some quasiperiodic variations, on time scales of years, have been detected through mean brightness variations, which are probably caused by magnetic cycles in the secondary, analogous to the sunspot cycle in the Sun (Applegate 1992, Warner 1988b). The most dramatic effect is seen when mass transfer from the secondary drops by a very large amount, sometimes for years, leading to a fall in mean brightness by several magnitudes as the accretion luminosity ceases. This is seen commonly in the polars, and has the advantage that the absorption spectrum of the primary can then be observed, and its magnetic field strength determined from Zeeman effect (these fields agree with those derived from the spacing of cyclotron harmonics observed in the spectra at high transfer rates: Wickramasinghe 1993). The cause of these low states is not yet understood. Similar low states are seen among some of the nova-like variables, where they have been attributed to deterministic chaos inherent in the feedback mechanism caused by heating of the secondary's atmosphere by the accretion luminosity (Wu, Wickramasinghe \& Warner 1995a).

In a certain range of mass transfer rate the accretion discs of non-magnetic systems have thermal instabilities. This is the result of there being two different equilibrium structures for an accretion disc - a consequence of the opacity of gas peaking near $\mathrm{T} \sim 10^{4} \mathrm{~K}$ so the requirements on opacity can be satisfied either in a high temperature convective disc or a low temperature disc. As the temperature of a disc is determined by the rate at which it must radiate the energy released by mass passing through it there are equilibrium structures for high and for low rates of mass transfer, but not for intermediate values (Hoshi 1979; Meyer \& Meyer-Hofmeister 1981). If the secondary provides such an intermediate value $\left(\sim 10^{13}-5 \times\right.$ $10^{16} \mathrm{gm} \mathrm{s}^{-1}$ ) no equilibrium is possible and the disc cycles between high temperature and low temperature states. This is the explanation of dwarf nova outbursts, where discs increase in luminosity by factors of 10-100 for a few days mostly on time scales of 10-100 d (with a few outbursting on time scales of $1-10 \mathrm{y}$ ).

Finally we must consider the effect of accumulation of hydrogen-rich material onto the surface of the primary. It has long been realised that this will lead to a thermonuclear runaway at the boundary of the degenerate core producing the classical nova eruption (Mestel 1952). For long it was thought that dwarf novae and classical novae are separate kinds of CVs, but it should be clear from the above discussion that all CVs must pass through the nova eruption stage, and that the present state and classification of a CV depends largely on its rate of mass transfer (and magnetic state). Indeed, in recent years connections between the various subclasses have been observed. After a nova eruption the system remains bright for decades, probably because of enhanced mass transfer caused by heating of the secondary's atmosphere by the white dwarf heated to $\sim 10^{5} \mathrm{~K}$ by the eruption. As the nova remnant cools so mass transfer from the secondary is reduced and the stable accretion disc changes to an unstable one - such dwarf nova behaviour has been seen long after eruptions in, e.g., GK Per V1017 Sgr and V446 Her (Honeycutt et al. 1998).

Nova eruptions in polars have a different consequence: interaction between the ejected shell and the secondary, exchanging orbital and shell angular momentum, with subsequent collapse of part of the temporary common envelope onto the primary, can break synchronicity of the primary's rotation. Nova Cyg 1975 (V1500 Cyg) has an orbital period of $0.1396 \mathrm{~d}$ and a primary rotation period (found from modulation of optical polarization) of $0.1372 \mathrm{~d}$. The latter is found to be increasing at a rate which will restore synchronism in $\sim 185 \mathrm{y}$ (Schmidt, Liebert \& Stockman 1995). This is very short compared with the intervals between eruptions (probably $10^{3}-10^{5} \mathrm{y}$ ), so V1500 Cyg spends most of its life as a polar. Other slightly desynchronized polars are BY Cam and RXJ 1940-1025, which we may deduce were prehistoric novae.

There must be a number of bright nova remnants unrecognised among the known nova-like variables: in this century several novae have arisen from stars found from archival plates to have had pre-nova brightnesses of $\sim 12-13 \mathrm{mag}$, brightnesses to which they eventually returned after a decade or so. Among the present known nova-likes, therefore, there must be nova remnants from past centuries, as well as some of the novae to occur in the next century. Surprisingly few novae were recognised and announced prior to 1850: there were only Nova Vulpecula 1670 and Nova Ophiuchi 1848 (some supernovae of course were observed, and 
some bright stars were observed and their positions measured without the observer being aware at the time that they were novae; Nova Sagittae 1783 was noticed but not proclaimed).

I will now select a few topics from the CV field in which recent advances in understanding have been achieved. These will illustrate the wide range of physical principles and processes that are brought to bear in this arena of astrophysical research.

\section{The Short-period Dwarf Novae: SU UMa stars}

A well defined subclass of dwarf novae, named after SU UMa, the first to be recognised, is observed to have a bimodal distribution of outburst behaviour: outbursts lasting $\sim 2$ days and less frequent $(>150 \mathrm{~d}$ ) outbursts that last for $\sim 20$ days and are about one magnitude brighter at maximum. The latter are known as superoutbursts. In a few SU UMa stars only superoutbursts occur; these are very infrequent, with recurrence times greater than a year (e.g. WZ Sge, which had superoutbursts in 1913, 1946 and 1978). There is strong observational evidence that a superoutburst is 'triggered' by an ordinary outburst - in the sense that an ordinary outburst is underway when the additional rise to supermaximum occurs.

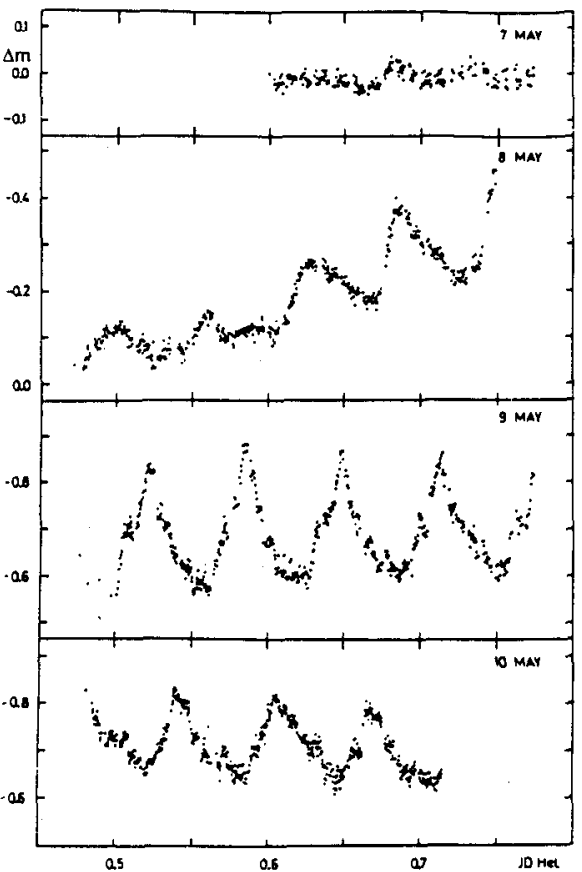

Fig. 3. Light curve of V436 Cen showing the growth of superhumps during a superoutburst From Semeniuk (1980).
A crucial clue to the understanding of the superoutburst phenomenon was the discovery of 'superhumps' during the maximal phase of the light curve (Vogt 1974; Warner 1975): all superoutbursts show brightness modulations with periods typically a few percent greater than the orbital period. The beat period between the superhump and orbital periods is usually $\sim 2 \mathrm{~d}$. After many attempts to explain this behaviour the solution emerged unexpectedly from multiple particle simulations of accretion discs (Whitehurst 1988): for sufficiently small mass ratio $\left(q=M_{2} / M_{1} \approx 0.25\right)$ accretion discs with large radii are perturbed into an elliptical form, the major axis of which precesses with the $\sim 2 \mathrm{~d}$ period. More complete simulations are able to reproduce the observed superhump modulations, which arise in tidal stresses in the disc, caused by passage of the secondary past the disc particles near apastron (Murray 1996).

From the geometry of Roche-lobe filing (semi-detached) binaries with main sequence secondaries, the requirement $q \widetilde{<} 0.25$ translates into a condition on the orbital period: $P_{\text {orh }}<3$ $\mathrm{h}$. This is compatible with the observation that all such short $P_{\text {orh }}$ dwarf novae are SU UMa stars.

During normal outbursts of dwarf novae the accretion disc expands in order to accept the angular momentum of gas falling onto the primary. For $q \widetilde{<} 0.25$ the $3: 1$ resonance of particle orbits with $P_{\text {orh }}$ is accessible to the outer edge of the disc. In normal outbursts of SU UMa stars only a small fraction of the disc mass is accreted on the primary, and the disc radius does not increase to the resonant radius. The gradual increase of mass and radius eventually results in a normal outburst in which the outer disc does reach resonance, and it is this that drives the disc into its elliptical shape and the consequent large tidal stress maintains the disc in a state of 
high viscosity until about half of its mass has drained onto the primary (Ichhikawa. Hirose \& Osaki 1989; Lubow 1991).

Recently a group of stars has been recognised as representing an extremum of the SU UMa type: the stars ER UMa, V1159 Ori, RZ Leo and DI UMa show normal outbursts at intervals of $\sim 4 \mathrm{~d}$ and superoutbursts at $\sim 50 \mathrm{~d}$. These must have mass transfer rates just marginally below what would result in stable discs (Warner 1995b). In the dwarf novae of longer orbital period, such high rates lead to the $Z$ Cam phenomenon, in which a slight increase of mass transfer out of the secondary causes a stable disc (i.e cessation of dwarf nova outbursts), keeping the star in an apparent outburst state for weeks or months. We may hope to see such a transition in one of these newly appreciated objects (they have been known for long as irregular variables, but ironically the rapidity with which they change state obscured their true nature. They are in a state of constant change - one normal outburst is not completed before the next begins, as a result they are of lower outburst range with no true quiescent state attained).

\section{Ultrashort-period Systems: the AM CVn Stars}

Among the variety of possible interactions of the stars in the initial binary system there are channels that lead (e.g. after two common envelope phases) to two helium cores revolving around each other. Two such degenerate stars can only be in a semi-detached state if their orbital period is very short. A few helium-rich stars showing brightness modulations on time scales of tens of minutes have been known for thirty years, but it is only recently that their light curves have been understood as ultrashort-period analogues of the more familiar hydrogen-rich CVs.

AM CVn (HZ 29) was found by Smak (1967) to have a double humped light curve with a period of $\sim 171 / 2 \mathrm{~min}$. High speed flickering indicated a mass-transferring binary (Warner \& Robinson 1972b). The modulation period is not strictly constant, which is now interpreted as being a superhump phenomenon: at such a short $P_{o r b}$ it is inevitable that $q \ll 0.25$ (the secondary mass $M_{2} \sim 0.09 \mathbf{M}_{\circ}$ ) and as the rate of mass transfer in AM CVn is quite high the accretion disc is maintained at a large radius and continually precessing (a few short $P_{u r h}$ systems with low amplitude permanent superhumps are also known among the H-rich CVs). The orbital modulation with a constant period of $1028.8 \mathrm{~s}$ has recently been detected (Harvey et al. 1998) and the disc precession period of $13.4 \mathrm{~h}$ has been observed spectroscopically from the asymmetry of line profiles that occur in a precessing elliptical disc (Patterson, Halpern \& Shambrook 1993).

Despite the unusual nature of the secondary (a very low mass degenerate helium star) the range of CV behaviour is shown among the AM CVn stars: AM CVn and EC15330-1403 are nova-like, with permanently high mass transfer; V803 Cen and CP Eri are nova-likes possessing states of low transfer rate; CR Boo is possibly a dwarf nova similar to VI159 Ori; and GP Com is in a low state which may eventually have a superoutburst (Warner 1995c).

The AM CVn stars found so far are bright $\left(\mathrm{m}_{\mathrm{v}} \sim 13-15\right)$ and mostly at high galactic latitude (where surveys for UV-rich stars have been made). There are therefore many more to be found. With their short $P_{\text {or }}$ and relative proximity $(\widetilde{<} 100 \mathrm{pc})$ the AM CVn stars should be apparently strong sources of gravitational radiation. They constitute an evolutionary channel leading ultimately to single $D B$ white dwarfs. No strongly magnetic helium systems have yet been identified.

\section{Secular and Cyclical Evolution of CVs}

Emerging from common envelope systems as detached binaries, evolving to become semidetached interacting binaries, continually reducing their secondaries' masses, expelling mass via nova eruptions (and steliar winds from the high state discs) - CVs clearly require a mechanism that extracts angular momentum from their orbits in order to drive the mass transfer process (for $q \widetilde{<} 1$, which is the case for almost all CVs, mass transfer from the secondary to the primary increases their orbital separation, so mass transfer is not a selfsustaining process) 
For the shortest period systems emission of gravitational radiation is an inevitable, and possibly the principal, angular momentum sink. At longer periods $\left(P_{o r b} \widetilde{<} 2 \mathrm{~h}\right.$ ) an additional process is required to explain the observed rates of transfer of $10^{-9}-10^{-8} \mathrm{M}_{0} \mathrm{y}^{-1}$. This has been identified as magnetic braking, in which the wind from the secondary, coupled to large radii onto its magnetic field lines, extracts angular momentum from the secondary which, being tidally locked in synchronism with the orbital revolution, ultimately extracts angular momentum from the orbit (Verbunt \& Zwaan 1981). Quantitative estimates of the strength of this process show that it is able to generate the observed mass transfer rates (Mestel \& Spruit 1987; McDermott \& Taam 1989; Tout \& Pringle 1992a).

The observed low space density of CVs having $2 \widetilde{<} P_{\text {orb }} \approx 3 \mathrm{~h}$ (the so-called orbital period gap) has been thought to be the result of a reduction of magnetic braking when the secondary is so reduced in mass that it becomes fully convective, changing the strength or topology of its magnetic field (Robinson et al. 1981) but it has recently been suggested (Clemens et al. 1998) that the expected change in shape of the mass-radius relation of mass sequence stars in the vicinity of $0.3 \mathrm{M}_{\circ}$ may suffice to explain the period gap: CVs race through the 2-3 h $P_{\text {orh }}$ range at a high transfer rate. Interestingly, CVs may as a result become the best objects for studying the internal structure of low mass main sequence stars.

When the mass of the secondary in $\mathrm{H}$-rich systems falls to $\sim 0.08 \mathbf{M}_{\odot}$ the secondary becomes degenerate and expands in radius as it further decreases in mass. The combination of mass transfer and orbital angular momentum loss then causes the orbital period to increase. For H-rich systems this occurs at $P_{\text {orh }} \cong 75 \mathrm{~min}$, which agrees well with the minimum $P_{\text {orb }}$ for such CVs. For helium systems the minimum is at $P_{\text {orb }} \sim 5 \mathrm{mins}$, no helium CVs have yet been found with such short periods - they will have high mass transfer rates and consequently short lifetimes near the minimum period.

Magnetic braking combined with gravitational radiation predicts that the mass transfer rate should be largely determined by $P_{o r b}$. This is strongly at variance with observation: at a given $P_{\text {orb }}$ there is a wide range of transfer rate (factors as large as $10^{3}$ or more). This manifests itself in the variety of $\mathrm{CV}$ subtypes that exist at any $P_{\text {orb }}$ : non-magnetic systems range from dwarf novae with very infrequent outbursts to highly luminous nova-likes with stable discs. It is clear that there is at least one other parameter that determines the current rate of transfer. It has been suggested (Livio \& Shara 1987) that nova eruptions heat the secondary and cause excess mass transfer for $\sim 100 \mathrm{y}$, with the result that the system must fall into a low state for long periods of time in order to maintain the secular mean transfer rate determined by the angular momentum sink. This would account for the apparent absence of CVs identifiable with ancient (Chinese and Korean) records of bright naked eye novae. The slow recovery from a low state (of 'hibernation') brings a CV up through the dwarf nova state to a stable disc before the next nova eruption. Such cyclical evolution (on time scales short compared with orbital evolution) may well happen, but the cause is not yet clear. Chaos inherent in the mass transfer irradiationenhanced process is a possible mechanism (Wu, Wickramasinghe \& Warner 1995b).

\section{Supersoft X-ray Sources}

A new type of $\mathrm{CV}$ has been observed, which fills a gap in the theoretical range of mass transfer possibilities. If a binary with $q>1$ reduces its separation (through magnetic braking) to the point where its secondary fills its Roche lobe, and if the secondary has a radiative envelope, then a stable high rate of mass transfer $\left(\sim 10^{-7} \mathrm{M}_{0} \mathrm{y}^{-1}\right)$ ensues. At such high rates of accretion stable nuclear burning occurs in the outer layers of a white dwarf, with a luminosity $\sim 10^{3} \mathrm{~L}_{0}$ and a flux peaking at an energy of $\sim 40 \mathrm{eV}$. A number of such 'supersoft' $X$-ray sources are known, and have been explained in the above manner (van den Heuvel et al. 1992; Kahabka \& van den Heuvel 1997). Steady nuclear burning also occurs in some post novae for up to a decade after the nova eruption (e.g. V1974 Cyg and GQ Mus).

\section{Properties of Accretion Discs}

In high state accretion discs - found in nova-likes, post nova eruption systems ('nova remnants') and dwarf novae near maximum of outburst - the observed flux comes from an almost two dimensional object. The (apparent) absolute magnitude is therefore sensitive to 
orbital inclination. This is most directly demonstrated in the nova remnants, where distances and hence absolute magnitudes are obtained from the eruption behaviour (the 'absolute magnitude - rate of decline relationship'): (apparent) absolute magnitudes are correlated with inclination in the way expected for a limb-darkened flat disc (Warner 1986a); this is confirmed by independent measurements of UV luminosities (Selvelli et al. 1990). This effect explains the existence of a few novae with anomalously large eruption amplitudes (e.g. DQ Her and T Aur: Payne-Gaposchkin 1957): these are systems of high inclination. Using this effect, DY Pup was predicted (Warner 1995a) and confirmed (Warner \& Van Zyl 1998) to be an eclipsing nova remnant.

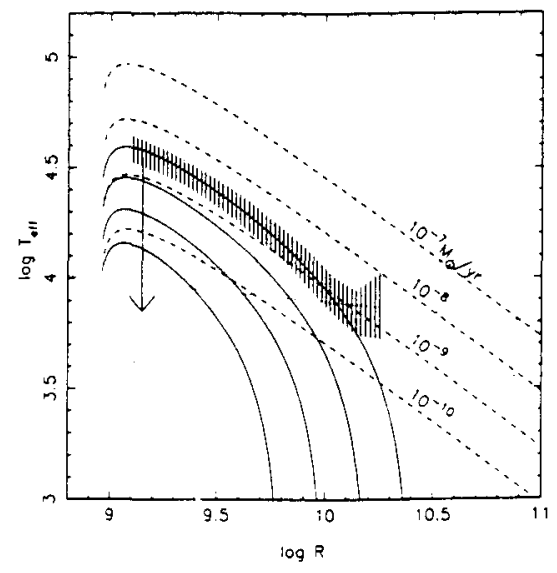

Fig. 4. Radial temperature distribution in the accretion disc of a dwarf nova during outburst. Dashed lines are theoretical distributions for non-steady discs at various rates of mass transfer. The hatched region is the observed distribution for $Z$ Cha. From Mineshige (1991).
In eclipsing CVs the tomographic technique introduced by Horne (1985) can be employed to map the intensity distribution over the surface of the accretion disc. Repeated in different wavelength bands a colour temperature distribution as a function of radial distance from the primary is deduced, which can be compared with the theoretical temperature distribution for steady state (Shakura \& Sunyaev 1973) or nonequilibrium (e.g. Mineshige \& Wood 1989) discs. There is general agreement for high state (e.g. Horne \& Cook 1985; Rutten et al. 1992) and low state discs (Mineshige \& Wood 1989). These analyses lead directly to measurement of the mass transfer rates (through the discs). typically $10^{13}-10^{16} \mathrm{gm} \mathrm{s}^{-1}$ in dwarf novae at

Using a tomographic restoration method on the emission line profiles of accretion disc spectra Marsh \& Horne (1988) show how to deduce the velocity distribution in the disc. From these Doppler maps Horne and Saar (1991) find an average intensity distribution $I(r) \widetilde{\propto} r^{-\frac{\tilde{2}}{2}}$ as a function of radial distance $r$ from the primary This relationship is what would be expected if the chromosphere of a disc is heated by process similar to those in cool stars, $i, . e$. the effects of a self-sustaining dynamo in the differentially rotating disc.

The identification of the mechanism by which sufficient effective viscosity is generated in a disc to account for the transition time scales observed in dwarf nova outbursts has long been a problem. Currently the most popular model is a dynamical instability that requires only very small magnetic fields initially to be present in the disc (Balbus \& Hawley 1991). This is capable of producing an unsteady self-sustaining dynamo with sufficient magnetically-generated viscosity (Tout \& Pringle 1992b).

Half of the potential energy of accreting gas is radiated away in the disc; in a simple model with a non-rotating primary the remaining half would be expected to be lost from the boundary layer (BL) that connects the inner edge of the disc to the primary. For low accretion rates this layer will be optically thin and BL shock temperatures $T \sim 10^{8} \mathrm{~K}$, emitting X-rays at $\sim 10 \mathrm{keV}$, should result. At high accretion rates the BL is optically thick and the thermalized radiation would emerge at $\mathrm{T} \sim 2 \times 10^{5} \mathrm{~K}$ (Pringle \& Savonije 1979). In fact, although hard X-rays are observed, and high mass transfer systems do have lower energy emission (e.g. Patterson \& Raymond 1985), the luminosities and temperatures of the latter systems are too low. From the ionization structure of winds from CV discs Hoare \& Drew (1991) find BL temperatures in the range $5 \times 10^{4}-1 \times 10^{5} \mathrm{~K}$. It remains uncertain why the BLs are of lower temperature and luminosity than the simple energy balance argument predicts. 


\section{Dwarf Nova Oscillations}

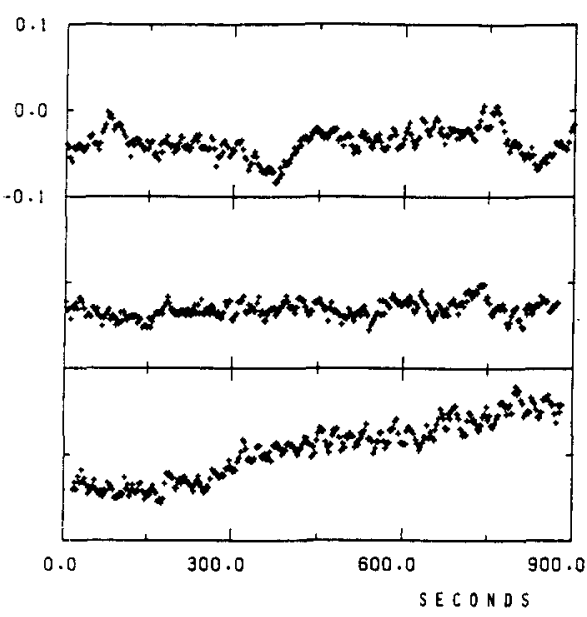

Fig. 5. Dwarf nova oscillations seen in the outburst light curve of TY PsA. From Warner, O'Donoghue \& Wargau (1989).
As an example of the unexpected or exotic phenomena that arise in CVs we can consider the short time scale periodic or quasiperiodic brightness modulations that occur in most dwarf novae during outburst, and spasmodically in some nova-likes. Discovered at very low amplitudes (seen rarely in the light curve with amplitudes $\sim 1 \%$; more generally only detectable by Fourier analysis with amplitudes as low as $0.02 \%$ ), dwarf nova oscillations (DNOs) have periods typically in the range 10-30 s (Warner and Robinson 1972a; Warner 1995a) with each star showing a small range. The periods change systematically during outburst, passing through a minimum value at maximum luminosity. The coherence of the oscillations also varies during outburst, being largest near maximum light but often showing good coherence for intervals of $1-2 \mathrm{~h}$ with abrupt small changes of period between. The DNOs are observed in SS Cyg, U Gem and VW Hyi in soft X-rays $(0.04-0.5 \mathrm{keV})(\mathrm{e} . \mathrm{g}$. Watson, King \& Heise 1985); these three CVs are

relatively near to the Earth, so soft X-ray emission is not totally absorbed by interstellar gas. DNOs have also been observed in the far UV (Mauche 1998).

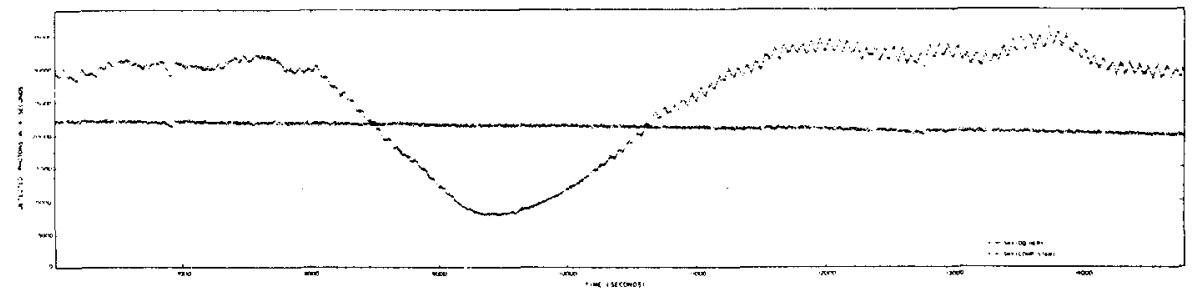

Fig. 6. Light curve of $D Q$ Her at 4 s time resolution showing eclipse of the $71 \mathrm{~s}$ oscillations. The horizontal light curve is that of a comparison star. From Nather (1978).

Less coherent modulations, known as quasi periodic oscillations (QPOs), are also observed in dwarf novae during outburst (Patterson, Robinson \& Nather 1977). These have periods a few times larger than the DNOs.

The origin of DNOs is not yet certain. A promising model invokes magnetically controlled accretion by relatively weak fields of the white dwarf primaries, the surface layers of which are spun up by enhanced accretion during dwarf nova outburst (Warner 1995d). The QPOs may be caused by non-radial oscillations in the accretion disc (Carroll et al. 1985).

An interesting practical use for DNOs arises from measurement of their phase variations during eclipse. As in the case of the 7ls oscillations in DQ Her, where the very high stability of the pulse enables phase shifts to be made with precision (Warner et al. 1972, Robinson 1998). phase shifts in HT Cas and UX UMa (Nather \& Robinson 1974; Patterson 1981) demonstrate that the whole accretion disc is emitting the DNO optical radiation: this is deduced to be reprocessed modulated hard radiation from the vicinity of the primary. 


\section{Intermediate Polars}

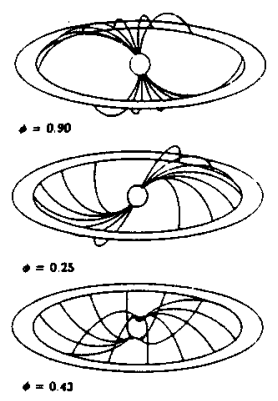

Fig. 7. Schematic view of accretion in an intermediate polar, viewed from three different rotational phases. From Hellier, Cropper \& Mason (1991)
The interaction of accretion disc plasma with the rotating magnetosphere of a white dwarf is currently beyond the realm of realistic computation. There is a wealth of observed phenomena awaiting quantitative interpretation; partial progress has been made in understanding some of these

Most IPs are probably close to a spin equilibrium where the torques acting on the primary through the field lines that connect it to parts of the disc that spin more rapidly or less rapidly (plus the material torque as accreting gas hits the primary) are in balance. From a theoretical evaluation of these torques (Ghosh \& Lamb 1979; Lamb 1989 ) it is possible to deduce the magnetic moment of the primary from its observed spin period and the accretion rate (found from estimating the total accretion luminosity, which is predominantly emitted at X-ray wavelengths). Such an approach gives surface field strengths $\sim 0.5-5$ $\times 10^{6} \mathrm{G}$ for the primaries in IPs. The large unpolarized

optical flux from the truncated accretion disc dilutes any cyclotron emission from the material accreting along field lines to the primary; nevertheless, circular polarization has been detected in the infrared of three IPs - presumably those with the largest field strengths - which indicate fields in the range $2-5 \times 10^{6} \mathrm{G}$ (Wickramasinghe, Wu \& Ferrario 1991; Buckley et al. 1997).

In the X-ray region IPs are both luminous and modulated: most IPs have been discovered as $\mathrm{X}$-ray sources. In general the modulation period is the spin period of the primary, caused by the $X$-ray emitting accretion regions being carried around as the primary rotates. There are usually two accretion regions, near the magnetic poles of the primary, rather like auroral arcs. The magnetic field axis is inclined to the rotation axis in all IPs so far studied; magnetic and rotational alignment would largely eliminate the spin modulation, but such an IP would still be a strong X-ray emitter.

Although there is strong evidence that the majority of IPs possess (truncated) accretion discs, it is possible to have a primary with field strong enough to prevent formation of a disc but too weak to ensure synchronous rotation. Then a process of 'discless accretion' occurs, with the accretion stream impacting directly onto the asynchronously rotating magnetosphere of the primary. This impact zone is another source of X-ray emission, but its modulation period is the synodic rather than the sidereal period of the primary. This situation may, however, be confused with that where stream material partially continues over the face of the disc and impacts onto the rotating magnetosphere. These cases may be distinguished by differences in modulation amplitude. Hellier (1992) finds most IPs to have discs, but Buckley et al. (1997) make a strong case for discless accretion in RXJ1712.6-2414.

The anisotropic hard radiation emitted by the asynchronously rotating primary periodically intercepts various parts of the binary that are revolving at the orbital period. These features may be the secondary star, the accretion stream or the disc thickening where it is impacted by the stream. Each of these features reprocesses the X-rays/UV into optical radiation, whose brightness may be modulated (through geometric or optical depth effects) on $P_{\text {orh }}$. This can lead not only to optical modulation at sidereal and synodic spin periods but also at other orbital sidebands of these spin periods (Warner 1986b). In some IPs the full set of predicted modulations is seen (e.g. FO Aqr: de Martino et al. 1994). The relative phases of these modulations can help to identify which component of the $\mathrm{CV}$ is reprocessing the hard radiation.

Changes of spin period on time scales of $\sim 10^{6} \mathrm{y}$ are observed, both spin-ups and spindowns occurring. These are to be expected of systems not perfectly in spin equilibrium - the present rotation period of the primary being determined by the average mass transfer rate over 
the past $10^{5}$ years, which is unlikely to match the current transfer rate from the secondary. (This is also the case in DQ Her, whose $71 \mathrm{~s}$ spin period is decreasing on a time scale $\sim 10^{5} \mathrm{y}$ as it attempts to find equilibrium with the high mass transfer rate of a recent post nova).

\section{Polars}
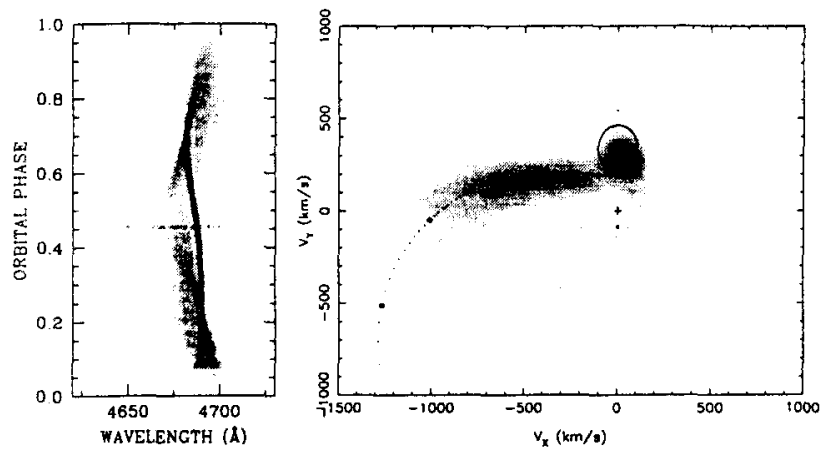

The power of doppler tomography to delineate the stream flow in strongly magnetic CVs is shown in Figure 8 . The initial timeresolved spectrum maps onto velocity coordinates and shows where the line emission is located - in HU Aqr clearly it is from the inter-star stream and from the side of the secondary pointing towards (and hence heated by) the primary and its accretion column

Fig. 8. Time resolved spectrum (left) and doppler tomogram (right) of the polar HU Aqr. The computed outline of the secondary star and the stream velocity trajectory are for a chosen set of parameters. From Schwope et al. (1995). (Schwope et al. 1995). The stream trajectory is a function of the mass ratio $q$; in the case of $\mathrm{HU}$ Aqr this

leads to $q=0.25$. Then from the observed duration of eclipse in this system, the inclination is reliably determined at $85^{\circ}$. In such eclipsing polars, therefore, some system parameters can be very well determined.

\section{Nova Eruptions}

The accumulation of hydrogen-rich material on the surface of a white dwarf leads ultimately to the pressure and temperature at the base of the layer reaching the point where nuclear reactions commence. As this is at a level where the gas is semi-degenerate a thermonuclear runaway ensues which ejects the gas accumulated since the previous eruption, and can also excavate part of the core of the white dwarf.

The duration between eruptions depends sensitively on the mass of the white dwarf: for masses approaching the Chandrasekhar limit the ejected mass is $\sim 10^{-6} \mathrm{M}_{\circ}$ and can occur on time scales of $\sim 100$ y (for mean mass transfer rates $\sim 10^{-8} \mathrm{M}_{\circ}, \mathrm{y}^{-1}$ ). This agrees with observations of recurrent novae. In general the recurrence time is $\sim 10^{3}-10^{5} \mathrm{y}$ and $\sim 10^{-4} \mathrm{M}_{\circ}$ is ejected. The greater frequency and energy of eruption in CVs of high mass biases mass determinations towards the high end of the range.

Total ejected shell masses are best determined from radio observations at $3-90 \mathrm{GHz}$, where the shell remains optically thin until 100-500 d after eruption (Hjellming 1990). Optical and infrared spectra of the shells typically show overabundances of CNO and sometimes $\mathrm{Ne}$ (Livio \& Truran 1992). This is caused either by mixing between the core and the bottom of the Hrich layer in the interval between eruptions, or to direct dredge-up during eruption (Kovetz \& Prialnik 1985).

Imaging of novae in the earliest stages of eruption has become possible in the radio region through the use of very long baseline interferometry (e.g. QU Vul: Taylor et al 1998) and at optical and UV wavelengths through the use of HST (e.g. Nova Cyg 1992: Paresce et al. 1995). The high resolution of HST also reveals remarkable detail in the dispersing shells of old novae (e.g. recurrent nova T Pyx: Shara et al. 1997). 


\section{Prospects}

Cataclysmic Variable stars, of which I have here given only a small example of their behaviour, will remain a growth industry for the foreseeable future. They are bright and populous in the $\mathrm{X}$-ray region, and so will be prominent in the surveys of the next generation of $\mathrm{X}$-ray satellites. The brightest quiescent CVs in the Magellanic Clouds are within reach of the new very large telescopes in the southern hemisphere; study of such groups of CVs at accurately known distances will reveal more of the multi-parameter evolutionary details of these systems. Furthermore, the brightest CVs remain the best objects for studies of steady and non-steady accretion discs, which will aid progess in understanding the discs now realised to be of great importance in a variety of other astrophysical objects.

\section{References}

Applegate, J.H., 1992. ApJ, 385, 621.

Balbus, S.A. \& Hawley, J.F., 1991. ApJ, 376, 214.

Buckley, D.A.H., et al., 1997. MNRAS, 287, 117.

Carroll, B.W., et al., 1985. ApJ, 266, 529.

Clemens, C. et al., 1998 . ApJ, 496, 352.

Crawford, J.A. \& Kraft, R.P., 1956. ApJ, 123, 44

Cropper, M.S., 1990. Sp. Sci. Rev. 54, 195.

de Martino, D., et al., 1994. Astr. Astrophys., 284, 125

Ghosh, P. \& Lamb, F.K., 1979. ApJ, 234, 296

Harvey, D.A. et al., 1998. ApJ Letts., 493, L105.

Hellier, C., 1992. MNRAS, 258, 578.

Hellier, C., Cropper, M.S. \& Mason, K. O., 1991. MNRAS, 248, 233.

Hjellming, R.M., 1990. Lect. Notes Phys. 364, 169.

Hoare, M.G. \& Drew, J.E. 1991. MNRAS, 249, 452.

Honeycutt, R.K et al., 1998. ApJ, 495, 933

Horne, K., 1985. MNRAS, 213, 129.

Horne, K. \& Cook, M.C., 1985. MNRAS, 214, 307.

Horne, K. \& Saar, S.H., 1991. ApJ, 374, L55.

Hoshi, R., 1979. Prog. Theor. Phys., 61, 1307.

Ichikawa, S., Hirose, M. \& Osaki, Y., 1993. Pub. astr. Soc. Japan, 45, 243.

Kahabka, P. \& van den Heuvel, E.P.J., 1997. Ann. Rev. Astr. Astrophys, 35, 69.

Kovetz, A. \& Prialnik, D., 1985. ApJ, 291, 812.

Kraft, R.P., 1961. ApJ, 134, 171.

Kraft, R.P., 1962. ApJ, 135, 408.

Kraft, R.P., 1964. ApJ, 139, 457.

Lamb, F.K., 1989, in Timing Neutron Stars, eds. H. Ogeiman \& E.P.J. van den Heuvel, Kluwer, p. 649.

Livio, M. \& Truran, J.W., 1992. ApJ, 389, 695.

Lubow, S., 1991. ApJ, 381, 259.

Marsh, T.R. \& Horne, K., 1988. MNRAS, 235, 269

Mauche, C., 1998. ApJ In press.

McDermott, P.N. \& Taam, R.E., 1989. ApJ, 342, 1019.

Meintjies, P.J. et al., 1994. ApJ, 434, 292.

Mestel, L., 1952. MNRAS, 112, 598

Mestel, L. \& Spruit, H.C., 1987. MNRAS, 226, 57.

Meyer, F. \& Meyer-Hofmeister, E., 1981. Astr. Astrophys., 104, L10

Mineshige, S., 1991. MNRAS, 250, 253.

Mineshige, S. \& Wood, J., 1989. MNRAS, 241, 259.

Murray, J, 1996. MNRAS, 279, 402.

Nather, R.E., 1978. PASP, 90, 477.

Nather, R.E. \& Robinson, E.L., 1974. ApJ, 190, 637.

Nather, R.E. \& Warner, B., 1971. MNRAS, 152, 209

Paczynski, B., 1976. Int. Astr. Union Symp. No. 73, p. 75 
Paresce, F. et al., 1995. Astr. Astrophys., 299, 823.

Patterson, J., 1981. ApJ Suppl., 45, 517.

Patterson, J. \& Raymond, J.C., 1985. ApJ, 292, 535.

Patterson, J, Halpern, J.P. \& Shambrook, A., 1993. ApJ, 419, 83.

Patterson, J., Robinson, E.L. \& Nather, R.E., 1977. ApJ, 214, 144.

Payne-Gaposchkin, C., 1957. The Galactic Novae, North-Holland, Amsterdam.

Robinson, E.L., et al., 1981. ApJ, 251, 611.

Robinson, E.L. et al., 1998. ApJ, In press.

Rutten, R.G.M. et al., 1992. Astr. Astrophys., 265, 159.

Schmidt, G.D., Liebert, J. \& Stockman, H.S., 1995. ApJ, 441, 414

Schwope, A.D. et al., 1995. Astr. Soc. Pacific Conf. Ser., 85, 166.

Selvelli, P.L. et al., 1990. IAU Colloq. No. 122, p. 65.

Semeniuk, I., 1980. Astr. Astrophys. Suppl., 39, 29.

Shakura, N.I. \& Sunyaev, R.A., 1973. Astr. Astrophys., 24, 337.

Shara, M.M. \& Prialnik, D., 1994. Astr. J., 107, 1542.

Shara, M.M. et al., 1997. Astr. J., 114, 258.

Smak, J., 1967. Acta Astr., 17, 255.

Tapia, S., 1977. ApJ, 212, L125.

Taylor, A.R. et al., 1998. Nature, 335, 235.

Tout, C.A. \& Pringle, J.E., 1992. MNRAS, 256, 269.

Tout, C.A \& Pringle, J., 1992b. MNRAS, 259, 604.

van den Heuvel, E.P.J. et al., 1992. Astr. Astrophys., 262, 97.

Verbunt, F. \& Zwaan, C., 1981. Astr. Astrophys., 100, L7.

Vogt, N., 1974. Astr. Astrophys., 36, 369.

Walker, M.F., 1956. ApJ, 123, 68.

Warner, B., 1975. MNRAS, 170, 219.

Warner, B., 1986a. MNRAS, 222, 11.

Warner, B., 1986b. MNRAS, 219, 347.

Warner, B., 1988a. High Speed Astronomical Photometry, Cambridge University Press.

Warner, B., 1988b. Nature, 336, 129.

Warner, B., 1995a. Cataclysmic Variable Stars, Cambridge University Press.

Warner, B., 1995b. Astrophys. Sp. Sci., 226, 187.

Warner, B., 1995c. Astrophys. Sp. Sci., 225, 249.

Warner, B., 1995d. Astr. Soc. Pacific Conf. Ser., 85, 343.

Warner, B. \& Robinson, E.L., 1972a. Nature Phys. Sci., 239, 2.

Warner, B. \& Robinson, E.L., 1972b. MNRAS, 159, 101

Warner, B. \& van Zyl, L., 1998. In preparation.

Warner, B., O'Donoghue, D. \& Wargau, W., 1989. MNRAS, 238, 73.

Warner, B. et al., 1972. MNRAS, 159, 321 .

Watson, M.G., King, A.R. \& Heise, J., 1985. Sp. Sci. Rev., 40, 127.

Whitehurst, R., 1988. MNRAS, 232, 35.

Wickramasinghe, D.T., 1993. in Cataclysmic Variables and Related Physics, eds. O. Regev \& G. Shaviv, Inst. Phys. Publ., Bristol, p. 213

Wickramasinghe, D.T., Wu, K \& Ferrario, L., 1991. MNRAS, 249, 460.

Wood, K.S., Imamura, J.N. \& Wolff, M.T., 1992. ApJ, 398, 593.

Wu, K., Wickramasinghe, D.T. \& Warner, B. 1995a. Astrophys. \& Sp. Sci. Library 205, 315.

Wu, K., Wickramasinghe, D. T. \& Warner, B., 1995b. Proc. Astr. Soc. Australia, 12, 60. 